\title{
Burnett function expansions with a bi-Maxwellian weight function for electron swarm physics
}

\author{
K. F. Ness ${ }^{1} \quad$ R. D. White ${ }^{2}$
}

(Received 31 August 2006; revised 29 June 2007)

\begin{abstract}
In the solution of Boltzmann's equation by polynomial expansion techniques it is important to choose the weight function as close as possible to the actual distribution function in order to ensure rapid convergence. In the case of electron motion through neutral gases in the presence of external electric and magnetic fields, the so-called moment method has had considerable success. The method is essentially a polynomial expansion of the electron velocity distribution function about a Maxwellian weight function at some arbitrary temperature. By choosing the temperature carefully in order to approximate the actual distribution adequate convergence can usually be obtained. However when the interactions between the electrons and the molecules is
\end{abstract}

See http://anziamj.austms.org.au/ojs/index.php/ANZIAMJ/article/view/123 for this article, (c) Austral. Mathematical Soc. 2007. Published July 10, 2007. ISSN 14468735 
'soft' and/or reactive processes cause a significant increase in the population of the high energy tail of the distribution function, convergence of the expansion rapidly deteriorates and may not be achieved. In this article we investigate the use of a bi-Maxwellian weight function to improve convergence by the use of a model interaction between the electrons and molecules. The idea being that a Maxwellian at the lower temperature should be sufficient to characterise the electrons in the bulk of the distribution, while a second Maxwellian of smaller amplitude but at a some what higher temperature is used to characterised the electrons in the high energy tail of the distribution.

\section{Contents}

1 Introduction

C219

2 Theory

C221

3 Results and discussion

C225

4 Conclusion

C228

References

C228

\section{Introduction}

A collection of charged particles moving through a background of neutral gas molecules under the influence of externally applied fields is referred to as a swarm. In the presence of an electric field the swarm particles gain energy from the field and are characterised by a temperature somewhat higher than the gas temperature. From a theoretical perspective the behaviour of the 
swarm can be described by the Boltzmann equation. Our theoretical understanding of swarms advanced considerably following the introduction of two temperature theories for solving the Boltzmann equation [10,3]. The two temperature theory is based upon a formal expansion of the electron or ion velocity distribution in a polynomial series around a suitably chosen Maxwellian (Gaussian) weight function. Prior to the introduction of two temperature theories, the expansion was done about a Maxwellian at the neutral gas temperature. This limited analysis to only weak fields. The name "two temperature" derives from the fact that the temperature of the Maxwellian weight function need not be the same as the neutral gas temperature. Although the introduction of the second temperature complicates the mathematics, the range of field strengths over which analysis can be done is considerably enhanced. Essentially the idea is that, by choosing the second temperature carefully, the weight function will be as close as possible to the actual ion/electron distribution and therefore convergence of the polynomial expansion will be rapid.

However, in some cases where the collision frequency decreases with energy, the convergence of the two temperature theory deteriorates and may fail $[8,4,9]$. In this situation it is believed that the high energy tail of the actual distribution function is decreasing slower than a Maxwellian. Skullerud [8] showed that the convergence of any polynomial series about a Maxwellian will fail in such a case. This led Ness and Viehland [6] to introduce a polynomial expansion about a bi-Maxwellian weight function. In this method, the weight function consists of the weighted sum of two Maxwellians. The lower temperature Maxwellian is used to represent the bulk of the ions/electrons, while a higher temperature Maxwellian in used to represent the more energetic particles in the tail of the distribution. The method proved successful in ion swarm physics, but it has not to date been applied to electron swarm physics, despite the fact that it may have proved useful in certain cases [5]. Although there are similarities in electron and ion swarms, there are important differences which impact on numerical solution of the Boltzmann equation. In particular, the electron-neutral mass 
ratios are around three orders of magnitude less than ion-neutral mass ratios. As a consequence, in colliding with the neutrals, the electron directions will be rapidly randomised, but they will lose little energy per collision, at least in the case of elastic collisions. Ions on the other hand will not have their directions so effectively randomised during collisions with the neutrals, rather they will tend to maintain the preferred direction of the acceleration due to the electric field. However, they will lose around half their energy per collision. Practically this means that electron swarm theories in general require fewer terms than ions swarm theories to account for anisotropy in velocity space, but they require considerably more terms in any expansion of the energy dependence.

In this article we apply the bi-Mawellian method to electron swarms. In the next section a summary of the theoretical aspects of the method is given. This is followed by the results for certain model interactions.

\section{Theory}

The motion of a dilute swarm of electrons moving through a background of much more dense neutral molecules in the presence of applied fields can be described by the linear Boltzmann equation

$$
\frac{\partial f}{\partial t}+\boldsymbol{c} \cdot \frac{\partial f}{\partial \boldsymbol{r}}+\boldsymbol{a} \cdot \frac{\partial f}{\partial \boldsymbol{c}}=-J\left(f, f_{0}\right),
$$

where $f(\boldsymbol{r}, \boldsymbol{c}, t)$ is the single-particle phase space distribution function, which is a function of position $\boldsymbol{r}$, velocity $\boldsymbol{c}$ and time $t$. The acceleration on an electron due to the external fields is denoted by $\boldsymbol{a}$. The collision operator $J\left(f, f_{0}\right)$ takes into account binary interaction between the electrons and neutral, where $f_{0}$ denotes the neutral molecule distribution function, which is assumed to be Maxwellian at the gas temperature $T_{0}$. In this article we consider only conservative collisions between the electrons and neutrals. Robson and Ness [7] give details of the collision operator. 
Equation (1) is a seven dimensional integro-differential equation which is solved for $f$. Once $f$ is obtained all quantities of interest describing the behaviour of the electron swarm can be determined. For the boundary free problem, the space-time dependence of $f$ is assumed to have the form

$$
f(\boldsymbol{r}, \boldsymbol{c}, t)=\sum_{j=0} f^{(j)}(\boldsymbol{c}) \cdot\left(-\frac{\partial}{\partial \boldsymbol{r}}\right)^{j} n(\boldsymbol{r}, t),
$$

where the $f^{(j)}$ are tensors of rank $j$, the dot denotes a $j$-fold scalar product and $n(\boldsymbol{r}, t)$ is the electron number density. Substitution of the hydrodynamic expansion (2) into equation (1) and equating coefficients of $(-\partial / \partial \boldsymbol{r})^{j} n$ results in a hierarchy of equations to solve for the velocity distribution functions $f^{(j)}(\boldsymbol{c})$, the first two members of which are [2]

$$
\begin{aligned}
& \boldsymbol{a} \cdot \frac{\partial f^{(0)}}{\partial \boldsymbol{c}}+J\left(f^{(0)}, f_{0}\right)=0, \\
& \boldsymbol{a} \cdot \frac{\partial f^{(1)}}{\partial \boldsymbol{c}}+J\left(f^{(1)}, f_{0}\right)=\boldsymbol{c} f^{(0)} .
\end{aligned}
$$

From each member of the hierarchy the various transport quantities can be calculated. For example, in the absence of reactive processes, $f^{(0)}$ yields the swarm mean energy $\langle\epsilon\rangle$ and the drift velocity $\boldsymbol{W}$, while $f^{(1)}$ yields the diffusion tensor $(\mathbf{D})[2]$;

$$
\begin{aligned}
\langle\epsilon\rangle & =\int \epsilon f^{(0)}(\boldsymbol{c}) d \boldsymbol{c}, \\
\boldsymbol{W} & =\int \boldsymbol{c} f^{(0)}(\boldsymbol{c}) d \boldsymbol{c}, \\
\mathbf{D} & =\int \boldsymbol{c} f^{(1)}(\boldsymbol{c}) d \boldsymbol{c} .
\end{aligned}
$$

In equation (5) $\epsilon=m c^{2} / 2$ is the kinetic energy of an electron.

In the moment method of solving any member of the above hierarchy the velocity dependence of the function $f^{(j)}$ is expanded in terms of Burnett 
functions,

$$
\phi_{m}^{[\nu l]}(\alpha \boldsymbol{c})=N_{\nu l}\left(\frac{\alpha c}{\sqrt{2}}\right)^{l} S_{l+1 / 2}^{(\nu)}\left(\frac{\alpha^{2} c^{2}}{2}\right) Y_{m}^{[l]}(\hat{\boldsymbol{c}})
$$

where

$$
\begin{aligned}
N_{\nu l}^{2} & =\frac{2 \pi^{2 / 3} \nu !}{\Gamma(\nu+l+3 / 2)}, \\
\alpha^{2} & =\frac{m}{k T_{b}},
\end{aligned}
$$

$S_{l+1 / 2}^{(\nu)}$ is a Sonine polynomial, and $Y_{m}^{[l]}(\hat{\boldsymbol{c}})$ is a spherical harmonic with $\hat{\boldsymbol{c}}$ denoting the polar angles of $\boldsymbol{c}$. The Burnett functions are the eigenfunctions of the collision operator for the case of constant collision frequency (Maxwell model) and are orthogonal with respect to a Maxwellian weight function, that is,

$$
\int w(\alpha, c) \phi_{m}^{(\nu l)}(\alpha \boldsymbol{c}) \phi_{m^{\prime}}^{\left[\nu^{\prime} l^{\prime}\right]}(\alpha \boldsymbol{c}) d \boldsymbol{c}=\delta_{\nu^{\prime} \nu} \delta_{l^{\prime} l} \delta_{m^{\prime} m}
$$

where

$$
w(\alpha, c)=\left(\frac{\alpha^{2}}{2 \pi}\right)^{3 / 2} \exp \left\{\frac{-\alpha^{2} c^{2}}{2}\right\} .
$$

Because the Burnett functions are orthogonal with respect to a Maxwellian function it is expedient to make the expansion

$$
f^{(j)}(\boldsymbol{c})=w(\alpha, c) \sum_{\nu=0}^{\infty} \sum_{l=0}^{\infty} \sum_{m=-l}^{l} F_{m}^{(\nu l)}(\alpha ; j) \phi_{m}^{[\nu l]}(\alpha \boldsymbol{c}) .
$$

In two temperature theories $\alpha$, that is, $T_{b}$ is chosen to optimise convergence of expansion (13). In the case of ions, $T_{b}$ is set to actual ion temperature [11], whereas for electron it is left as a free parameter [3].

In the case of a bi-Maxwellian weight function the following expansion is made

$$
f^{(j)}(\boldsymbol{c})=\left[b w\left(\alpha_{1}, c\right)+(1-b) w\left(\alpha_{2}\right)\right] \sum_{\nu=0}^{\infty} \sum_{l=0}^{\infty} \sum_{m=-l}^{l} F_{m}^{(\nu l)}(\alpha ; j) \phi_{m}^{[\nu l]}(\alpha \boldsymbol{c}),
$$


where

$$
\begin{aligned}
\alpha_{1}^{2} & =\frac{m}{k T_{1}}, \\
\alpha_{2}^{2} & =\frac{m}{k T_{2}},
\end{aligned}
$$

and $0 \leq b \leq 1$. In equation (14) $\alpha$ may or may not be related to $\alpha_{1}$ and $\alpha_{2}$. Substitution of expansion (14) into equations (3) and (4), premultiplying by $\phi_{m^{\prime}}^{\left(\nu^{\prime} l^{\prime}\right)}$ and integrating over all velocities transforms each member of the hierarchy into a matrix equation which then can be solved for the expansion coefficients (moments) $F_{m}^{(\nu l)}(\alpha ; j)$. In deriving these matrix equations we use the orthonormal property (11) and the relationship [1]

$$
\begin{aligned}
\phi_{m}^{(\nu l)}(q \boldsymbol{c})=[\nu ! & \Gamma(\nu+l+3 / 2)]^{1 / 2} q^{l} \\
& \times \sum_{k=0}^{n} \frac{q^{2(n-k)}\left(1-q^{2}\right)^{k}}{k !\left[(n-k) ! \Gamma(n-k-l+3 / 2)^{1 / 2}\right]} \phi_{m}^{(\nu-k l)}(\boldsymbol{c}),
\end{aligned}
$$

where $q$ is a scalar. Once the expansion coefficients are obtained, the transport coefficients and other quantities of interest can be obtained. If an electric field $\boldsymbol{E}$ is the only external field present then the drift velocity has only one component $W$, that is parallel to $\boldsymbol{E}$ and the diffusion tensor has two components, one parallel $D_{L}$ and the other perpendicular $D_{T}$ to $\boldsymbol{E}$.

Practical application of either expansion (13) or expansion (14) requires truncation of both the $l$ and the $\nu$ indices to finite values, $l_{\max }$ and $\nu_{\max }$, say. The choice of $l_{\max }$ and $\nu_{\max }$ depends upon the convergence requirements and/or the time limit set for computation. The greater the value of $l_{\max }$ required for convergence, the greater the anisotropy of the distribution function in velocity space. The greater the value of $\nu_{\max }$ required for convergence the greater the variation of the energy dependence of the distribution function from the weight function. In the next section, both expansions (13) and (14) are applied to a simple elastic model and convergence of the transport coefficients in the polynomial index $\nu$ are compared. 


\section{Results and discussion}

Here we present results for a model situation where only elastic collisions occur. The following model was considered:

$$
\begin{aligned}
& \sigma(\epsilon)=\epsilon^{-3 / 4} \AA^{2} \\
& m_{0}=4 \mathrm{amu}, \quad T_{0}=293 \mathrm{~K} \\
& E / N=0.2 \mathrm{Td}, \quad 1 \mathrm{Td}=10^{-21} \mathrm{Vm}^{2},
\end{aligned}
$$

where $\sigma(\epsilon)$ denotes the cross section for collisions between the electrons and neutrals of impact energy $\epsilon$, and $m_{0}$ is the mass of the neutral particles. In swarm physics the transport processes are controlled by the ratio of electric field strength to the neutral gas number density, that is, $E / N$. The Townsend $(\mathrm{Td})$ is the accepted unit of $E / N$. The above model represents a collision frequency decreasing with energy. As $E / N$ increases, convergence of an expansion about a single Maxwellian weight function becomes increasingly harder to achieve for this model. The value of $E / N$ chosen $(0.2 \mathrm{Td})$ is close to the limit for convergence of the two temperature theory.

In Figures 1 and 2 the convergence of the transport coefficients is shown as a function of the $\nu$ index for an expansion about a single Maxwellian weight function for four value of $T_{b}$. For this model $l_{\max }$ was set to 3 ; however, convergence to four figures was achieved by $l=1$. All four values of $T_{b}$ are necessarily somewhat larger than the actual electron temperature of $6,150 \mathrm{~K}$. In situations where the collision frequency decreases with energy, $T_{b}$ must be chosen larger than the actual electron temperature if convergence in the $\nu$ index is to be achieved. This example gives an indication of how much larger this must be. In this case only the convergence of the swarm mean energy can be deemed satisfactory. The converged value of the transport coefficients, after 60 Sonine polynomials, for each $T_{b}$ is indicated on the various curves in Figures 1 and 2. As we proceed from the drift velocity to diffusion perpendicular to $\boldsymbol{E}$ to diffusion parallel to $\boldsymbol{E}$ the convergence deteriorates. In particular, we notice that the converged value appears dependent upon 

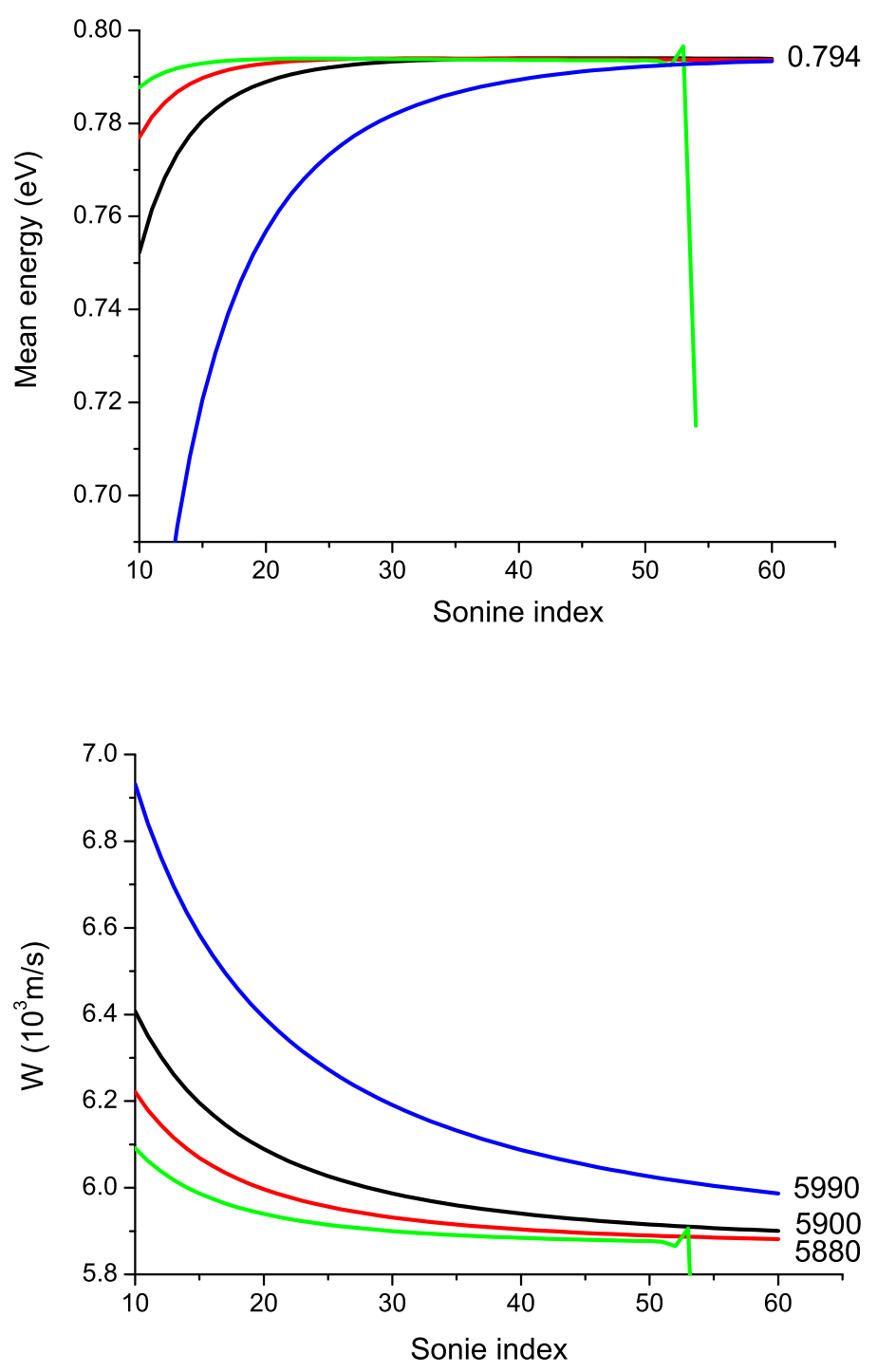

Figure 1: Convergence of the mean energy and drift velocity in the $\nu$ index for single Maxwellian weight functions. (Colour: $T_{b}$ ): (Green: $50000 \mathrm{~K}$, ), (Red: 70000K), (Black: 100000K), (Blue: 200000K). 

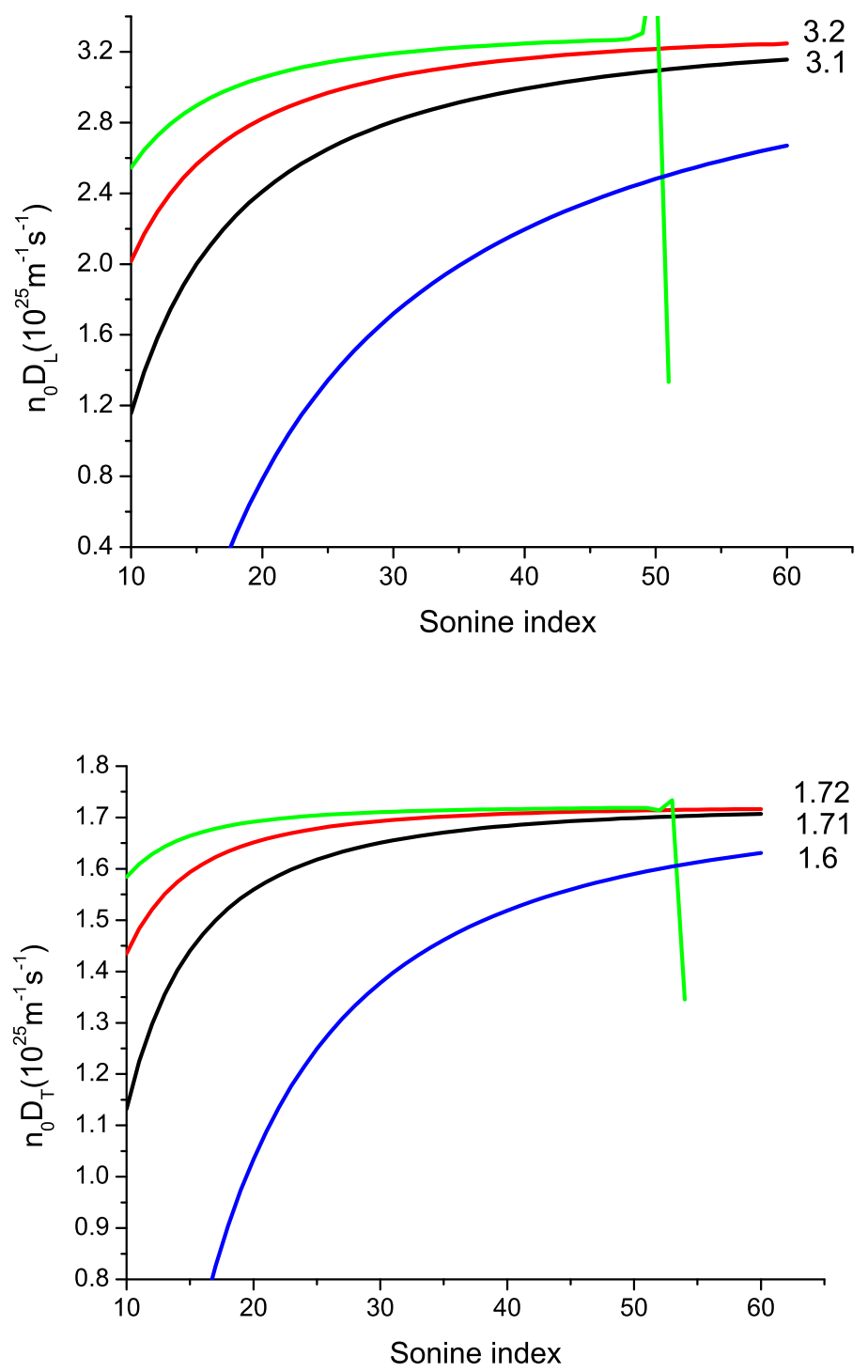

Figure 2: Convergence of the diffusion coefficients in the $\nu$ index for single Maxwellian weight functions. (Colour: $T_{b}$ ): (Green: $\left.50000 \mathrm{~K},\right)$, (Red: $70000 \mathrm{~K})$, (Black: $100000 \mathrm{~K})$, (Blue: $200000 \mathrm{~K})$. 
the choice of $T_{b}$. This of course should not be the case. This behaviour is typical in situations where the interaction between the electron and neutral are 'soft'.

In Figures 3 and 4, the convergence of the transport coefficients is shown as a function of the $\nu$ index for expansions about four different bi-Maxwellian weight functions, the value of $T_{1}, T_{2}$ and $b$ are indicated on the figures. For all transport coefficients shown convergence to three or four figure is achieved between 30 to 40 terms in the Sonine polynomial expansion. This is considerably better than that for the single Maxwellian weight function.

\section{Conclusion}

An outline of the motivation and theory behind an expansion of the electron velocity distribution function about a bi-Maxwellian weight function has been presented. The theory was applied to a particular model where it was known that convergence problems for the two temperature (expansion about a Maxwellian) theory would be encountered. For this particular model the success of the bi-Maxwellian expansions was demonstrated. The next step is to apply the technique to real gases, for example, electron transport in water vapour/fluorine, where it is known that the interactions between the electron and neutrals are 'soft'.

\section{References}

[1] J. Aisbett, J. M. Blatt and A. H. Opie, "General calculation of collision integral for linearised Boltzmann transport equation", J. Stat. Phys. 11 (1974) 4441. C224 

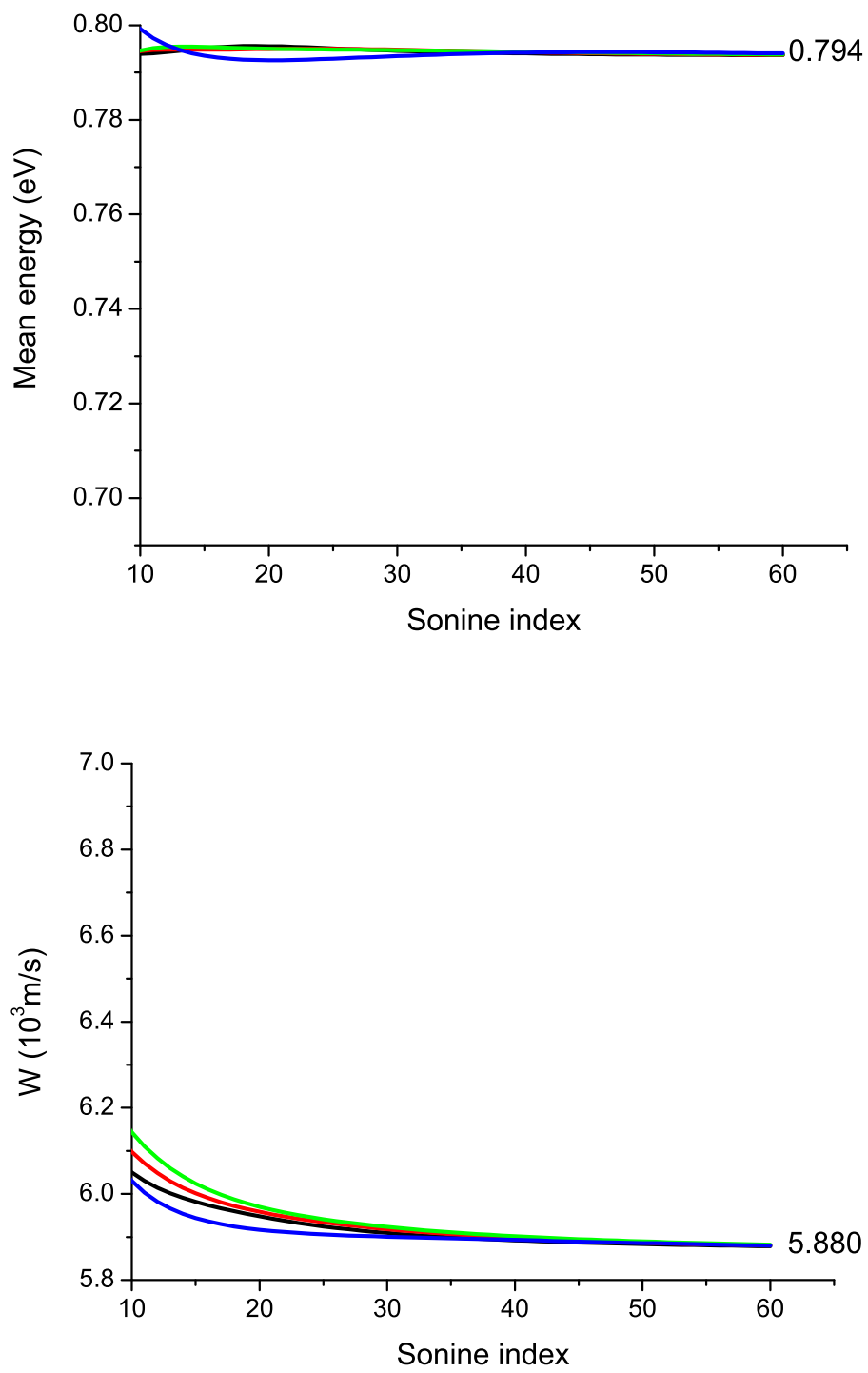

Figure 3: Convergence of the mean energy and drift velocity in the $\nu$ index for bi-Maxwellian weight functions. ((Colour: $\left.T_{1}, T_{2}, b\right)$ : (Green: 10000,100000,0.5), (Red: 10000,100000,0.7), (Black: 10000,100000,0.9), (Blue: 6000,100000,0.5). 

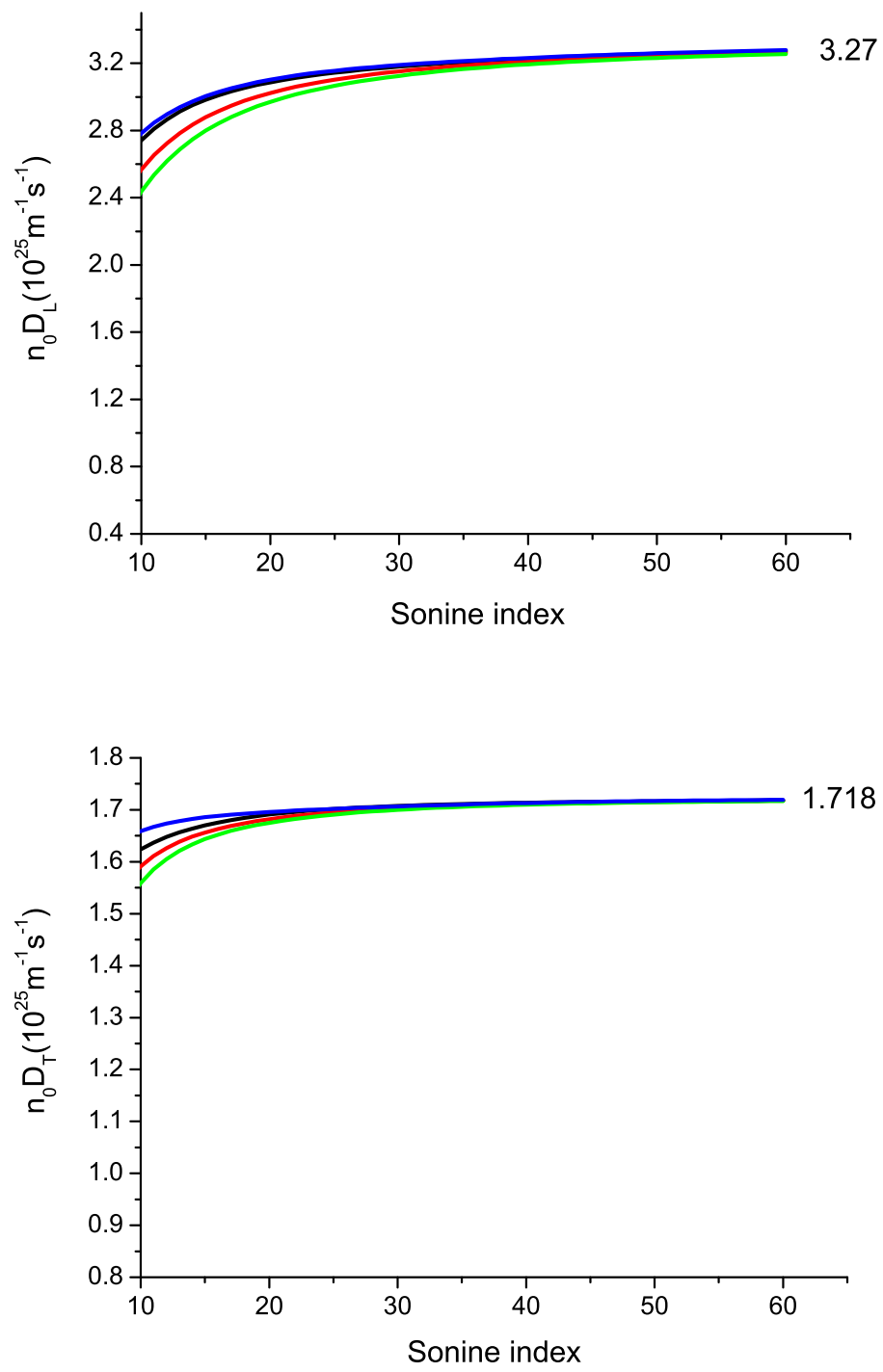

Figure 4: Convergence of the diffusion coefficients in the $\nu$ index for bi-Maxwellian weight functions. (Colour: $T_{1}, T_{2}, b$ ): (Green: 10000,100000,0.5), (Red: 10000,100000,0.7), (Black: 10000,100000,0.9), (Blue: 6000,100000,0.5). 
[2] K. Kumar, H. R. Skullerud and R. E. Robson, "Kinetic-theory of charged-particle swarms in neutral gases", Aust. J. Phys. 33 (1980) 343. C222

[3] S. L. Lin, R. E. Robson and E. A. Mason, "Moment theory of electron-drift and diffusion in neutral gases in an electrostatic field", J. Chem. Phys 71 (1979) 3483. C220, C223

[4] K. F. Ness and R. E. Robson, "Velocity distribution function and transport-coefficients of electron swarms in gases. 2. Moment equations and applications", Phys. Rev. A 34 (1986) 2185. C220

[5] K. F. Ness and R. E. Robson, "Transport-properties of electrons in water-vapour" Phys. Rev. A 38 (1988) 1446. C220

[6] K. F. Ness and L. A. Viehland, "Distribution-functions and transport-coefficients for atomic ions in dilute gases", Chem. Phys. 148 (1990) 225. C220

[7] R. E. Robson and K. F. Ness, "Velocity distribution function and transport-coefficients of electron swarms in gases: Spherical harmonics decomposition of the Boltzmann equation", Phys. Rev. A 33 (3) (March 1986) 2068. C221

[8] H. Skullerud, "On the calculation of ion swarm properties by velocity moment methods", J. Phys. D 17 (1984) 913. C220

[9] L. A. Viehland, "Velocity distribution-functions and transport-coefficients of atomic ions in atomic gases by a Gram-Charlier approach", Chem. Phys. 179 (1994) 71. C220

[10] L. A. Viehland and E. A. Mason, "Gaseous ion mobility in electric-fields of arbitrary strength", Ann. Phys. 91 (1975) 499. C220

[11] L. A. Viehland and E. A. Mason, "Gaseous ion mobility and diffusion in electric-fields of arbitrary strength", Ann. Phys. 110 (1978) 287. C223 


\section{Author addresses}

1. K. F. Ness, School of Mathematics, Physics \& Information Technology, James Cook University, Townsville, Australia. mailto:Kevin.Ness@jcu.edu. au

2. R. D. White, School of Mathematics, Physics \& Information Technology, James Cook University, Townsville, Australia. 